\title{
Molecular Characterization and Geographic Distribution of a Mymonavirus in the Population of Botrytis cinerea
}

\author{
Fangmin Hao ${ }^{1,2}$, Mingde $\mathrm{Wu}^{1,2, *(1)}$ and Guoqing $\mathrm{Li}^{1,2}$ \\ 1 The State Key Laboratory of Agricultural Microbiology, Huazhong Agricultural University, \\ Wuhan 430070, China; haofangmin@163.com (F.H.); guoqingli@mail.hzau.edu.cn (G.L.) \\ 2 The Key Laboratory of Plant Pathology of Hubei Province, Huazhong Agricultural University, \\ Wuhan 430070, China \\ * Correspondence: mingde@mail.hzau.edu.cn
}

Received: 24 July 2018; Accepted: 14 August 2018; Published: 15 August 2018

\begin{abstract}
Here, we characterized a negative single-stranded (-ss)RNA mycovirus, Botrytis cinerea mymonavirus 1 (BcMyV1), isolated from the phytopathogenic fungus Botrytis cinerea. The genome of $\mathrm{BcMyV1}$ is $7863 \mathrm{nt}$ in length, possessing three open reading frames (ORF1-3). The ORF1 encodes a large polypeptide containing a conserved mononegaviral RNA-dependent RNA polymerase (RdRp) domain showing homology to the protein L of mymonaviruses, whereas the possible functions of the remaining two ORFs are still unknown. The internal cDNA sequence (10-7829) of BcMyV1 was 97.9\% identical to the full-length cDNA sequence of Sclerotinia sclerotiorum negative stranded RNA virus 7 (SsNSRV7), a virus-like contig obtained from Sclerotinia sclerotiorum metatranscriptomes, indicating BcMyV1 should be a strain of SsNSRV7. Phylogenetic analysis based on RdRp domains showed that BcMyV1 was clustered with the viruses in the family Mymonaviridae, suggesting it is a member of Mymonaviridae. BcMyV1 may be widely distributed in regions where B. cinerea occurs in China and even over the world, although it infected only $0.8 \%$ of tested B. cinerea strains.
\end{abstract}

Keywords: Botrytis cinerea; Botrytis cinerea mymonavirus 1; Mymonaviridae

\section{Introduction}

Botrytis spp., a group of widespread plant pathogenic fungi, can infect more than 1400 plant species, causing gray mold disease on many economically important crops [1]. Besides being pathogens of many plants, Botrytis spp. are also ideal hosts for viruses. Among sequenced Botrytis viruses, most positive single-stranded (+ss)RNA viruses were classified into five families-Alphaflexiviridae, Gammaflexiviridae, Hypoviridae, Narnaviridae, and a recent proposed family Fusariviridae-while most double-stranded (ds)RNA viruses were assigned into three families-Endornaviridae, Partitiviridae, and Totiviridae - and the genus Botybirnavirus [2-5]. In addition, a few sequenced Botrytis viruses, including Botrytis cinerea RNA virus 1 [6], Botrytis ourmia-like virus [7], and Botrytis cinerea negative-stranded RNA virus 1 (BcNSRV1) [8], remained unclassified.

Compared with +ssRNA and dsRNA viruses, $(-\mathrm{ss}) \mathrm{RNA}$ viruses are rarely reported in Botrytis cinerea as well as in other fungi [1]. Mononegaviruses are a group of nonsegmented (-ss)RNA viruses with the genomes of 8.9-19 kb in length, although there are some exceptions [9]. Most reported mononegaviruses infect invertebrates, vertebrates, and plants, whereas only few have been shown to infect fungi [10-13]. Mononegaviruses are divided into eight families-Bornaviridae, Filoviridae, Paramyxoviridae, Rhabdoviridae, Pneumoviridae, Sunviridae, Nyamiviridae, and Mymonaviridae [12]—of which Mymonaviridae (genus Sclerotimonavirus, type species Sclerotinia sclerotiorum negative-stranded RNA 
virus 1 (SsNSRV1)) is a newly established viral family that accommodates mononegaviruses infecting fungi in the order Mononegavirales [14]. In addition, eight other viruses/virus-like contigs, including soybean leaf-associated negative-stranded RNA viruses 1-4 (SlaNSRV1-4), Sclerotinia sclerotiorum negative stranded viruses 2-4 (SsNSRV2-4), and Fusarium graminearum negative-stranded RNA virus 1 (FgNSRV1), are phylogenetically closer to SsNSRV1, and may also belong to Mymonaviridae [15-17].

In the present study, we describe the genome of a (-ss)RNA virus infecting the fungus $B$. cinerea, namely Botrytis cinerea mymonavirus 1 (BcMyV1). Genomic and phylogenetic analysis indicates that $\mathrm{BcMyV1}$ was most closely related to Sclerotinia sclerotiorum negative-stranded RNA virus 7 (SsNSRV7) [18] and also showed homology to other fungal mononegaviruses. In addition, we also determined the incidence and geographic distribution of $\mathrm{BcMyV} 1$ in the population of $B$. cinerea in China.

\section{Materials and Methods}

\subsection{Fungal Strains, Culture Conditions, and Biological Characterization}

B. cinerea strains Ecan17-2 was originally obtained through single conidium isolation from diseased oilseed rape (Brassica napus) stem in Shiyan, Hubei Province, China, and strain B05.10 of B. cinerea was used as a control [3]. In addition, $508 \mathrm{~B}$. cinerea strains from 40 counties/cities in 11 provinces of China were used for testing the presence of $\mathrm{BcMyV1}$. All strains were stored at $4{ }^{\circ} \mathrm{C}$ and working culture was established [19]. The mycelial growth was determined on potato dextrose agar (PDA) in petri dishes [3], while the pathogenicity of B. cinerea strains was determined on detached Nicotiana benthamiana leaves $[3,19,20]$.

\section{2. dsRNA Extraction and Purification}

dsRNAs from B. cinerea mycelia was extracted and purified as described previously [20] and was further confirmed based on resistance to DNase I and S1 nuclease (Promega, Madison, WI, USA). The extracted dsRNA was fractionated by agarose gel $(1 \%, w / v)$ electrophoresis and visualized by staining with ethidium bromide $(1.5 \mu \mathrm{g} / \mathrm{L})$ and viewing on a UV transilluminator.

\section{3. cDNA Cloning and Sequencing}

After separation by agarose gel electrophoresis, the dsRNA segment (BcMyV1 replication intermediates, approximately $10 \mathrm{~kb}$ in size based on the DNA marker) was gel-purified by using AxyPrepTM DNA Gel Extraction Kit (Axygen Scientific, Inc.; Union City, CA, USA) as described by Wu et al. [21]. The cDNAs of BcMyV1 were produced using a random-primer-mediated PCR amplification protocol [6] and were then sequenced [21]. The terminal sequences of the dsRNA were cloned through ligating the $3^{\prime}$-terminus for each strand of the dsRNA with the $5^{\prime}$-terminus of the 110A adaptor (Table S1) using T4 RNA ligase (Promega Corporation, 2800 Woods Hollow Road, Madison, WI, USA) at $16{ }^{\circ} \mathrm{C}$ for $18 \mathrm{~h}$ and were then reverse transcribed using primer RC110A (Table S1). The cDNA strands were then used as template for PCR amplification of the $5^{\prime}$ - and $3^{\prime}$-terminal sequences with the primer RC110A and the corresponding sequence specific primers (Figure S1 and Table S1). Cloning of the $3^{\prime}$ - or $5^{\prime}$-terminal sequences of the dsRNA was performed for three rounds. In addition, the internal region of the BcMyV1 genome was also amplified through RT-PCR with four sequence-specific primer pairs (Figure S1 and Table S1). All these amplicons were detected by agarose gel electrophoresis, gel-purified, and cloned into Escherichia coli $\mathrm{DH} 5 \alpha$ and sequenced as previously described [19]. All cDNA sequences were assembled to obtain the full-length cDNA sequence of BcMyV1.

\subsection{Sequence Analysis}

Open reading frames (ORFs) in the full-length cDNA sequences of BcMyV1 in strain Ecan17-2 of $B$. cinerea were deduced using the ORF Finder program on the website of the National Center for Biotechnology Information (NCBI, http:/ / www.ncbi.nlm.nih.gov/gorf/gorf.html). The homologous 
sequences searching for the full-length cDNA sequences and deduced polypeptides of BcMyV1 were carried out at the NCBI database by using the BlastN and BlastP programs, respectively. CDD database (http://www.ncbi.nlm.nih.gov/Structure/cdd/wrpsb.cgi) searching predicted the domains present in the polypeptide sequence. Multiple alignments of the sequences of mononegaviral RNA-dependent RNA polymerase (RdRp) domains in the polypeptides encoded by BcMyV1 and other mononegaviruses were performed using the ClustalW program in MEGA 7.0 [22]. Phylogenetic trees based on the sequences of RdRp domains were constructed using the neighbor-joining (NJ) method and tested with a bootstrap of 1000 replicates to ascertain the reliability of a given branch pattern in MEGA 7.0. Putative transmembrane helices sequences were predicted using the TMHMM server version 2.0 (http://www.cbs.dtu.dk/services/TMHMM/) [23].

\subsection{Detection of BcMyV1 in B. cinerea Population}

The total RNAs of 508 B. cinerea strains were extracted using the TRIzol ${ }^{\circledR}$ reagent (Invitrogen Corp, Carlsbad, CA, USA) as described previously [19], and the presence of BcMyV1 was determined by using RT-PCR with primer pairs M-RT-F/R (Table S1), which was designed to amplify a specific band of $728 \mathrm{bp}$ in size.

\section{Results}

\subsection{B. cinerea Strain Ecan17-2 Exhibits Hypovirulence Traits}

After cultivation on a PDA plate for 9 days, strain Ecan17-2 formed colonies with no production of sclerotia, whereas strain B05.10 produced massive sclerotia on the colony (Figure 1A). In addition, the radial mycelial growth of Ecan17-2 on PDA, averaging $2.9 \mathrm{~mm} /$ day, was significantly slower than that of strain B05.10 (14.8 mm/day). The virulence assay on detached $N$. benthamiana leaves revealed that the average lesion diameter $(6.9 \mathrm{~mm})$ caused by strain Ecan $17-2$ was significantly smaller than that $(29.3 \mathrm{~mm})$ of strain B05.10 (Figure 1B).
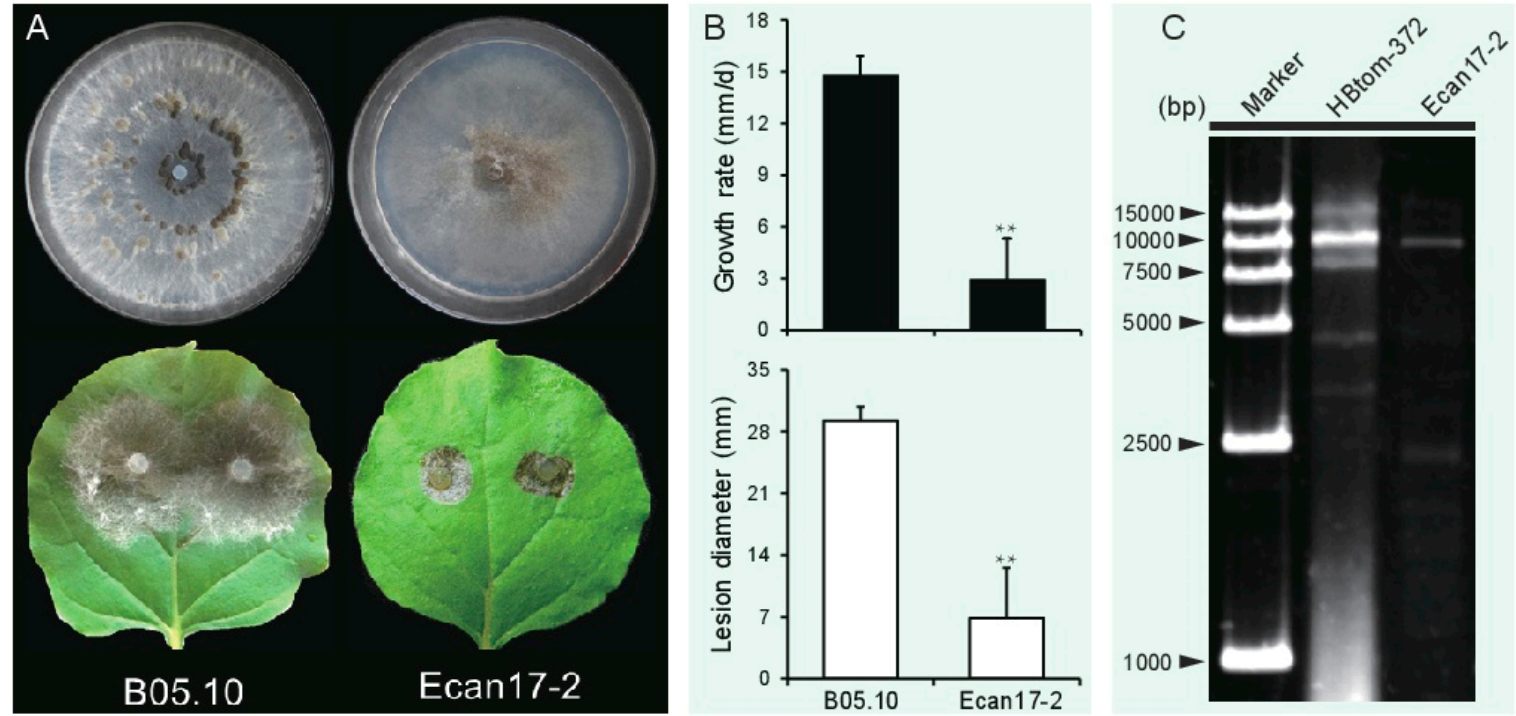

Figure 1. (A) Culture morphology (upper, $20{ }^{\circ} \mathrm{C}, 9$ days) and pathogenicity assay (lower, $20^{\circ} \mathrm{C}$, 3 days) of Botrytis cinerea strains Ecan17-2 and B05.10 on potato dextrose agar (PDA) and detached $N$. benthamiana leaves, respectively. (B) Radial mycelial growth rate $\left(20^{\circ} \mathrm{C}\right.$, upper) on PDA and lesion diameter $\left(20^{\circ} \mathrm{C}, 72 \mathrm{~h}\right.$, lower) on detached $N$. benthamiana leaves of strains Ecan $17-2$ and B05.10. "*** indicates a significant difference $(p<0.01)$ between strains Ecan17-2 and B05.10 in both pathogenicity and radial mycelial growth rate. (C) Agarose gel electrophoresis of dsRNAs extracted from the mycelia of B. cinerea strains Ecan17-2 and HBtom-372. 


\subsection{Genome Analysis of BcMyV1}

After DNase I and S1 nuclease digestion, a major dsRNA segment was detected through electrophoresis in the mycelium of B. cinerea strain Ecan17-2 with the size of approximately $10.0 \mathrm{~kb}$, which was slightly smaller than the dsRNA-B (Botrytis cinerea fusarivirus 1 (BcFV1), $8411 \mathrm{bp}$ ) detected in strain HBtom-372 [3]. The coding strand (GenBank accession no. MH648611) of BcMyV1 was $7863 \mathrm{nt}$ long, with a GC content of $41.6 \%$, possessing three ORFs (ORF1-3) and two short untranslated regions (UTRs) of $76 \mathrm{nt}$ and $472 \mathrm{nt}$ in length at the $5^{\prime}$ - and $3^{\prime}$-terminus, respectively. The ORF1 was predicted to encode a putative large polypeptide of 1968 amino acid (aa) residues (Figure 2A), which contains a putative mononegaviral RdRp domain and a mononegaviral mRNA-capping region $\mathrm{V}$ (Figure $2 \mathrm{~A}$ ). The ORF2 and ORF3 encoded two proteins of 169 aa and 250 aa in length, respectively. In addition, the $21 \mathrm{nt}$ long repeated sequence $3^{\prime}$-UAAAUUUCUUUGAUCCUCUAU-5' was detected in the two UTRs between the three ORFs (Figure 2B).

The results of the Blast search showed that the nucleotide sequence of the internal region (10-7829) of the BcMyV1 genome was $97.9 \%$ identical to the full-length nucleotide sequence of a contig obtained from metatranscriptomes of Sclerotinia sclerotiorum isolates, SsNSRV7 [18]. In addition, the polypeptide encoded by BcMyV1 ORF1 was almost 99.9\% identical to the protein L of SsNSRV7 and also showed homology to the protein L of SsNSRV1 (33.1\% aa identity), FgNSRV1 (33.8\% aa identity), and several (-ss)RNA viruses identified through deep sequencing (Table 1). Four conserved motifs, I-IV, found in mononegaviruses were also identified in the protein L encoded by BcMyV1 ORF1 (Figure 2C). Unlike SsNSRV1, transmembrane (TM) domains (Figure S2) were found at the C-proximal protein L of BcMyV1. However, the proteins encoded by ORF2 and ORF3 showed no significant sequence similarity with proteins in the database of NCBI by using BlastP search.

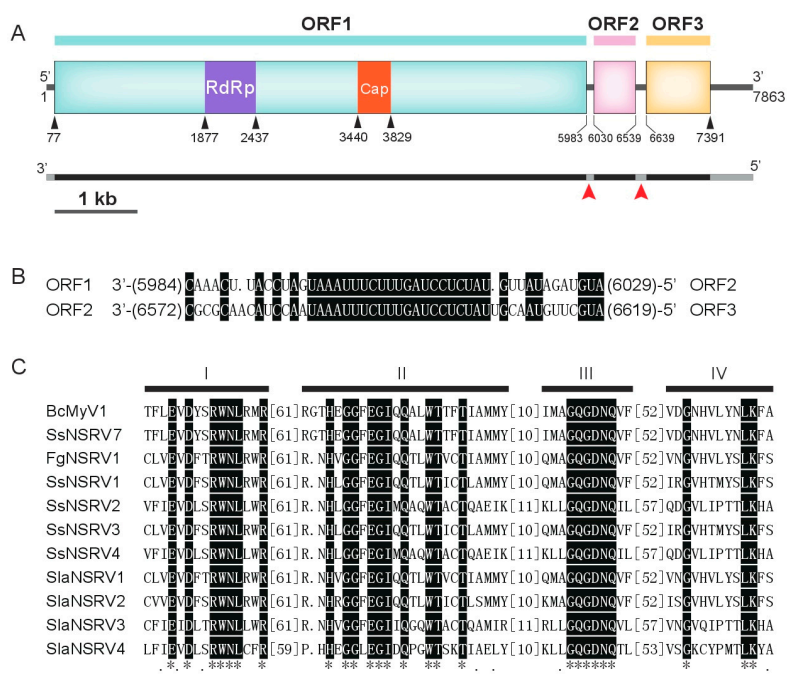

Figure 2. (A) Schematic diagram of the genome organization of Botrytis cinerea mymonavirus 1 (BcMyV1). The coding strand of BcMyV1 is $7863 \mathrm{nt}$ long and contains three Open reading frames (ORFs), and the ORF1 encode a protein L of 1968 amino acids (aa), possessing a mononegaviral RNA-dependent RNA polymerase (RdRp) domain and a mRNA-capping region V (Cap) domain. ORF2 and ORF3 encode two proteins of 169 aa and 250 aa, respectively. The black bars indicate the coding regions, and the gray bars represent the untranslated regions (UTRs) on the genome of BcMyV1. Two red arrowheads point out the positions of a $21 \mathrm{nt}$ repeat region on the two UTRs, and the detailed sequence information are listed in (B). The numbers in the parentheses indicate the nt positions nearby the parentheses. (C) Multiple alignment of the amino acid sequences of RdRp in the protein L encoded by BcMyV1 and other (-ss)RNA viruses. "** indicates identical amino acid residues; and "." indicate low chemically similar amino acid residues. The abbreviations of virus names are listed in Table 1. 


\subsection{Phylogenetic Analysis of BcMyV1}

To define the phylogenetic relationship of BcMyV1 with other viruses in Mononegavirales (Table 1), a phylogenetic tree was established based on the mononegaviral RdRp domain. BcMyV1 firstly formed a tight cluster with SsNSRV7 and then clustered with (-ss)RNA mycoviruses from S. sclerotiorum, F. graminearum, and other viral-like contigs, forming an independent clade of Mymonaviridae with the bootstrap support of $99 \%$. In addition, other viruses from Bornaviridae, Sunviridae, Filoviridae, Rhabdoviridae, Paramyxoviridae, Pneumoviridae, and Nyamiviridae also formed the corresponding viral family clades (Figure 3). Therefore, we suppose that BcMyV1 should be a member in the virial family Mymonaviridae.

Table 1. Percentage of sequence identities between Botrytis cinerea mymonavirus 1 and other mononegaviruses according to the multiple alignments of the full-length protein $\mathrm{L}$ and the RNA-dependent RNA polymerase domain.

\begin{tabular}{|c|c|c|c|c|c|}
\hline \multirow{2}{*}{ Family } & \multirow{2}{*}{ Virus } & \multirow{2}{*}{ Acronym } & \multicolumn{2}{|c|}{ aa Identity (\%) } & \multirow{2}{*}{ Accession no } \\
\hline & & & Full Sequence & RdRp & \\
\hline \multirow{10}{*}{ Mymonaviridae } & $\begin{array}{c}\text { Sclerotinia sclerotiorum } \\
\text { negative-stranded RNA virus } 7\end{array}$ & SsNSRV7 & 99.85 & 100 & MF444285 \\
\hline & $\begin{array}{c}\text { Sclerotinia sclerotiorum } \\
\text { negative-stranded RNA virus } 1\end{array}$ & SsNSRV1 & 33.12 & 56.68 & NC_025383.1 \\
\hline & $\begin{array}{c}\text { Sclerotinia sclerotiorum } \\
\text { negative-stranded RNA virus } 2\end{array}$ & SsNSRV2 & 22.28 & 41.45 & KP900931.1 \\
\hline & $\begin{array}{c}\text { Sclerotinia sclerotiorum } \\
\text { negative-stranded RNA virus } 3\end{array}$ & SsNSRV3 & 33.57 & 56.15 & NC_026732.1 \\
\hline & $\begin{array}{c}\text { Sclerotinia sclerotiorum } \\
\text { negative-stranded RNA virus } 4\end{array}$ & SsNSRV4 & 21.67 & 39.38 & KP900930.1 \\
\hline & $\begin{array}{c}\text { Soybean leaf-associated } \\
\text { negative-stranded RNA virus } 1\end{array}$ & SlaNSRV1 & 32.75 & 59.36 & KT598225.1 \\
\hline & $\begin{array}{c}\text { Soybean leaf-associated } \\
\text { negative-stranded RNA virus } 2\end{array}$ & SlaNSRV2 & 33.12 & 61.5 & KT598227.1 \\
\hline & $\begin{array}{c}\text { Soybean leaf-associated } \\
\text { negative-stranded RNA virus } 3\end{array}$ & SlaNSRV3 & 21.43 & 37.31 & KT598228.1 \\
\hline & $\begin{array}{c}\text { Soybean leaf-associated } \\
\text { negative-stranded RNA virus } 4\end{array}$ & SlaNSRV4 & 17.82 & 34.57 & KT598229.1 \\
\hline & $\begin{array}{l}\text { Fusarium graminearum } \\
\text { negative-stranded RNA virus } 1\end{array}$ & FgNSRV1 & 32.75 & 59.36 & MF276904.1 \\
\hline \multirow{4}{*}{ Bornaviridae } & Jungle carpet python virus & JCPV & 14.84 & 24.6 & MF135780 \\
\hline & Southwest carpet python virus & SWCPV & 14.33 & 21.93 & MF135781 \\
\hline & Loveridge's garter snake virus 1 & LGSV1 & 14.28 & 23.53 & KM114265 \\
\hline & Variegated squirrel bornavirus 1 & VSBV1 & 14.16 & 25.13 & LN713681 \\
\hline \multirow{2}{*}{ Rhabdoviridae } & Rabies virus & RabV & 14.97 & 23.62 & AB517659 \\
\hline & Maize mosaic virus & MMV & 15.19 & 23.12 & NC_005975.1 \\
\hline \multirow{2}{*}{ Paramyxoviridae } & Newcastle disease virus & NDV & 14.94 & 27.69 & JF827026.1 \\
\hline & Measles virus & MV & 15.44 & 30.77 & NC_001498.1 \\
\hline \multirow{2}{*}{ Nyamiviridae } & Midway nyavirus & MIDMV & 15.81 & 26.42 & NC_012702.1 \\
\hline & Nyamanini nyavirus & NYMV & 16.01 & 26.42 & NC_012703.1 \\
\hline \multirow{2}{*}{ Filoviridae } & Rose rosette virus & RRV & 10.57 & 11.4 & HQ871942 \\
\hline & Raspberry leaf blotch virus & RLBV & 10.71 & 10.27 & FR823299 \\
\hline Sunviridae & Reptile sunshinevirus 1 & RSV-1 & 14.26 & 24.26 & NC_025345 \\
\hline \multirow{2}{*}{ Pneumoviridae } & $\begin{array}{c}\text { Human respiratory syncytial } \\
\text { virus }\end{array}$ & HRSV & 13.43 & 22.68 & NC_001781 \\
\hline & Pneumonia virus of mice & PVM & 14.22 & 22.8 & NC_006579 \\
\hline
\end{tabular}




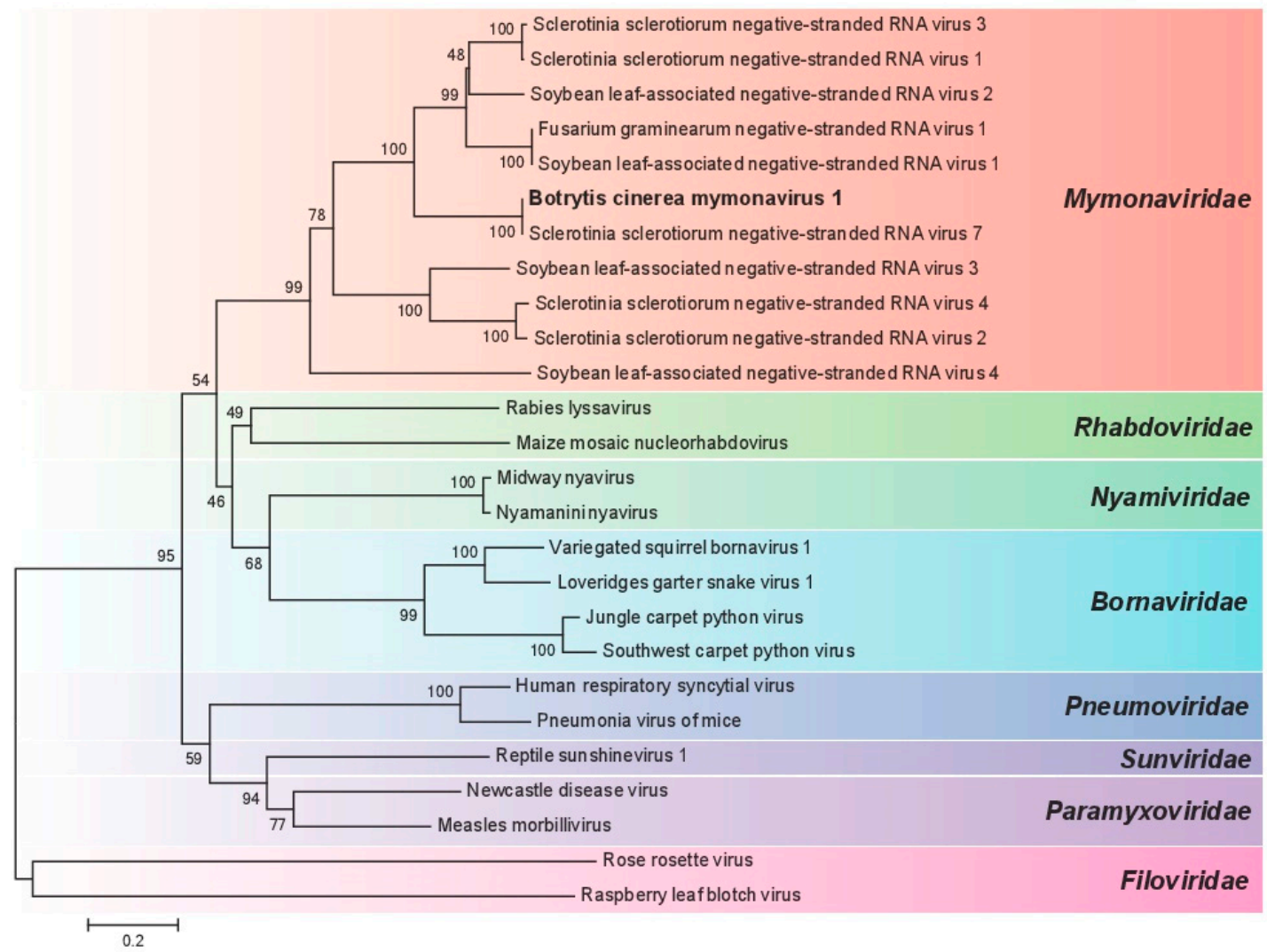

Figure 3. Phylogenetic analysis of Botrytis cinerea mymonavirus 1 (BcMyV1) based on RdRp domain from strain Ecan17-2 of B. cinerea.

\subsection{Incidence and Distribution of BcMyV1}

In order to investigate the incidence and distribution of BcMyV1 in China, 508 B. cinerea strains from China were tested for the presence of BcMyV1 by using RT-PCR with the primer pair M-RT-F/R (Table S1). BcMyV1 infection was detected in only 4 out of the $508(0.8 \%)$ tested B. cinerea strains (Figures 4 and 5.). In these BcMyV1-infected strains, Bs6-23 and Bs6-33 were collected from the same location (Beijing, China), whereas strain JLaub-11 was collected from Changchun of Jilin Province.

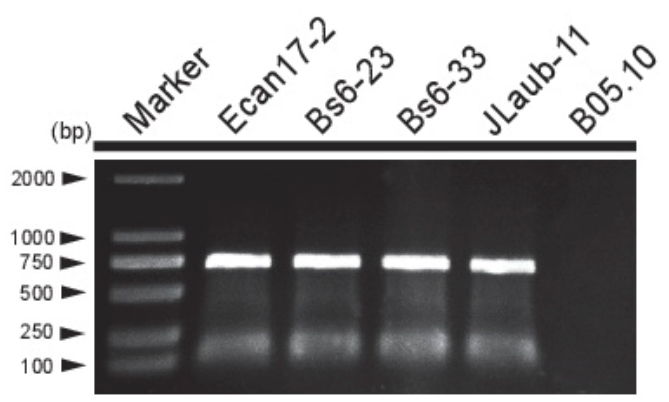

Figure 4. RT-PCR detection of Botrytis cinerea mymonavirus 1 (BcMyV1) in five B. cinerea strains. Strains Ecan17-2 and B05.10 served as positive and negative controls, respectively. 


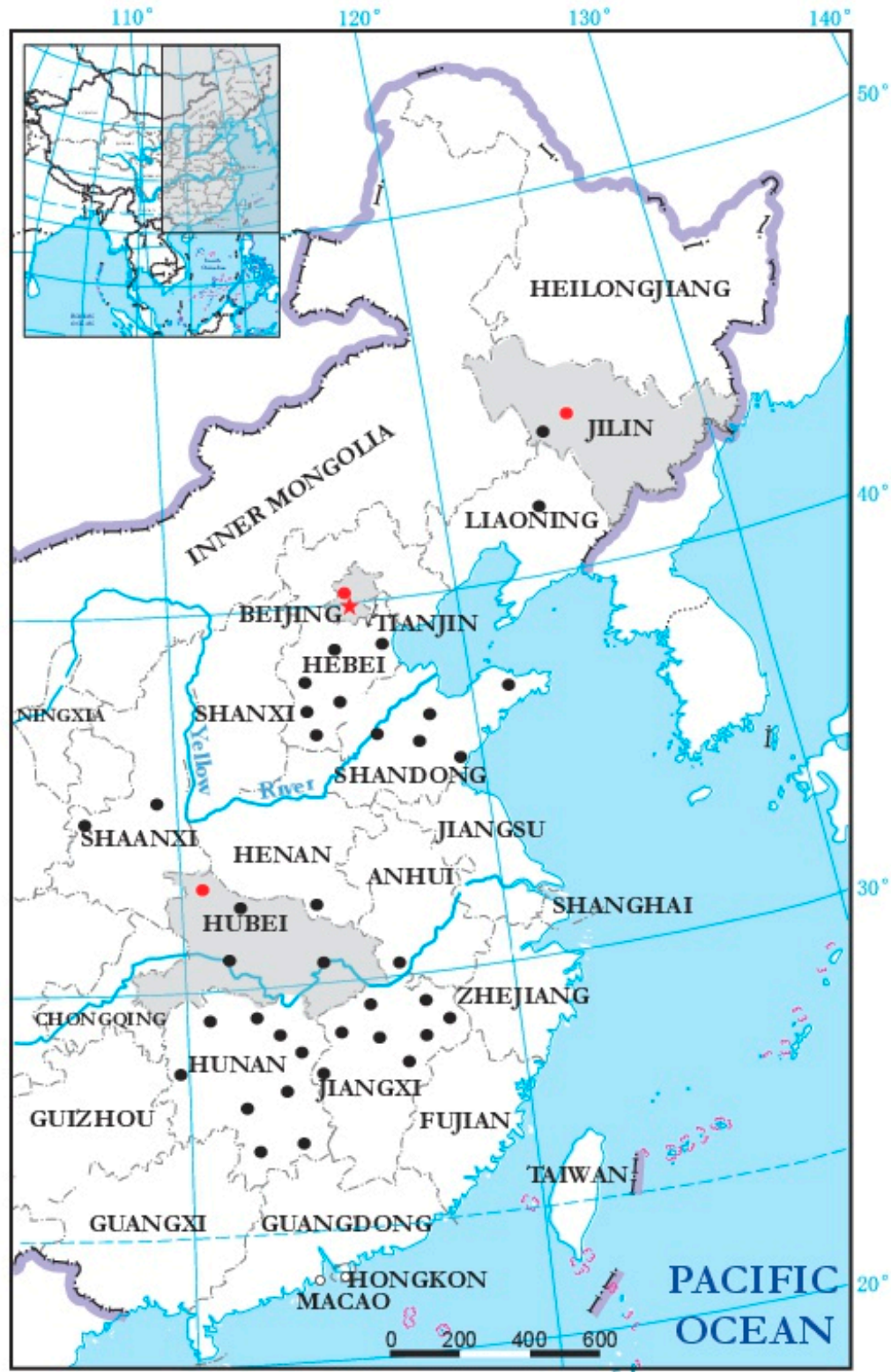

Figure 5. Geographic distribution of Botrytis cinerea mymonavirus 1 (BcMyV1) in 11 provinces of China. The black dots indicate the places where BcMyV1 was not detected, whereas places where $\mathrm{BcMyV1}$ was detected are indicated as red dots and the corresponding provinces are also highlighted in grey on the map.

\section{Discussion}

In the present study, we characterized the genome of a (-ss)RNA mycovirus, namely, BcMyV1, infecting the hypovirulent strain Ecan17-2 of B. cinerea. Notwithstanding that numerous mycoviruses have been reported in $B$. cinerea, only one case of a (-ss)RNA virus (BcNSRV1) had been characterized [8]. BcNSRV1 is phylogenetically related to members of the genus Emaravirus in the viral family Bunyaviridae. However, phylogenetic analysis based on RdRp domain indicated that BcMyV1 should belong to the viral family Mymonaviridae in the order Mononegavirales. As high sequence similarity was observed between BcMyV1 and SsNSRV7, BcMyV1 should be a strain of SsNSRV7 [18]. Nonetheless, the nucleotide sequence of BcMyV1 was longer at both $5^{\prime}$ - and $3^{\prime}$-termini than those of SsNSRV7, indicating the sequence of SsNSRV7 in the NCBI database might be incomplete. 
In S. sclerotiorum, SsNSRV1 infection was closely related to the debilitation symptoms of the infected S. sclerotiorum strain, including slow growth on PDA, loss of the ability to produce sclerotia, and pathogenicity on oilseed rape [14]. Similarly, B. cinerea strain Ecan17-2 carrying BcMyV1 also displayed reduced mycelial growth on PDA and attenuated virulence on $N$. benthamiana, indicating possible negative effects of BcMyV1 on its host. However, a faint dsRNA segment of approximately $2.4 \mathrm{~kb}$ in length (Figure 1C) was also detected in strain Ecan17-2, indicating coinfection of other viruses or defective/satellite RNAs with BcMyV1 [3]. Thus, sequencing the $2.4 \mathrm{~kb}$ dsRNA in the traditional way is warranted to ascertain the causal agent of hypovirulence in B. cinerea strain Ecan17-2. Moreover, deep sequencing [8] may also be an option to determine the full view of the viral infection in strain Ecan17-2. Generally, two aspects of approach have been used for construction of isogenic lines that aim to elucidate the effects of mycoviral infection on their hosts. Firstly, viruses could be introduced into virus-free strains by using the techniques like pairing-culture [3], virion transfection [21], and construction of infectious cDNA clones [24]. On the other hand, some investigations, including sequential hyphal tip isolation [25], protoplasts/small mycelial fragments regeneration [26], treatment of cycloheximide [27,28], or cAMP-rifamycin [29], and single spore isolation [20] were also explored to cure the viruses in their original strains. Therefore, similar experiments will also be carried out to elucidate the role of $\mathrm{BcMyV} 1$ on B. cinerea biology in the future.

It is of interest that BcMyV1 was detected in two different fungal species, B. cinerea and S. sclerotiorum. Although the same mycovirus is rarely detected in different fungi, there are still a few exceptions. In addition to the present case, another mycovirus, Botrytis porri botybirnavirus 1 (BpBV1), was also detected in both B. porri and S. sclerotiorum $[18,21]$. We suppose that viral interspecific transmission may frequently occur between B. cinerea and S. sclerotiorum, although they belong to different genera. Some factors may increase the possibility of viral transmission between the two species. Firstly, B. cinerea is a close relative of S. sclerotiorum, and their genes share $83 \%$ aa identity on average between the two fungi [30]. Thus, viruses may adapt a new host more easily when viral transmission occurs from one host to the other. Secondly, both fungi have a broad host range [1,31], and many plant species are hosts for both B. cinerea and S. sclerotiorum. Therefore, the contact between the two fungi may frequently occur in small niches under field conditions [30]. Thirdly, viral interspecies transmission through anastomosis has also been reported in a few cases, including from Aspergillus niger to A. nidulans [32], from S. sclerotiorum to S. minor [33], and from Cryphonectria parasitica to $C$. nitschkei [34], suggesting that viral interspecies transmission by anastomosis between $B$. cinerea and S. sclerotiorum might also be possible. Finally, recent studies have shown that insects and mites are also potential vectors during mycoviral transmission [35,36], and similar mechanisms may increase the possible viral transmission between B. cinerea and S. sclerotiorum as well.

Although -ssRNA viruses have been reported in several fungal species [8,14-18], the information of their incidence and distribution remain unclear. Moreover, investigation of other BcMyV1-infected $B$. cinerea strains may also help us to uncover their effects on $B$. cinerea. On the contrary, unlike strain Ecan17-2, two strains of B. cinerea (Bs6-33 and JLaub-11) carrying BcMyV1 grew quickly and produced massive sclerotia, and only one strain, Bs6-23, showed similar culture morphology to that of strain Ecan17-2 (data not shown). This suggests that more complex interactions may exist between BcMyV1 and the $B$. cinerea population. Therefore, genome-wide association study $[37,38]$ may be helpful to further elucidate the role of genetic variation in the response of $B$. cinerea to viral infection. Compared with other viruses infecting B. cinerea, including Botrytis cinerea endornavirus 1 [2], Botrytis cinerea hypovirus 1, BcFV1 [3], Botrytis cinerea mitovirus 1 [39], Botrytis virus F, and Botrytis virus X [5], the incidence of BcMyV1 in the Chinese B. cinerea population was very low, accounting for only $0.8 \%$ in the tested strains. The linear distance between Beijing and Shiyan is over $900 \mathrm{~km}$, and Changchun is over $800 \mathrm{~km}$ away from Beijing. These results indicate that BcMyV1 might be widely distributed in regions where $B$. cinerea occurs of China, although the infection rate is low. This may be one reason why (-ss)RNA viruses were rarely reported in the population of $B$. cinerea. Despite the low incidence, BcMyV1 still had a wide geographic distribution and may not be only limited to China, 
as the homologous virus-like contig was obtained from S. sclerotiorum strains in Australia. This suggests BcMyV1 may have a global distribution.

Supplementary Materials: The following are available online at http:/ / www.mdpi.com/1999-4915/10/8/432/s1 Table S1: Oligonucleotide primers/adaptor used in this study. Figure S1: Schematic representation of the strategy used for full cDNA sequence cloning of Botrytis cinerea mymonavirus 1 (BcMyV1). Figure S2: Transmembrane domains prediction for ORF1 encoded polypeptide L of BcMyV1.

Author Contributions: F.H., M.W., and G.L. conceived and designed the experiments. F.H. performed the experiments; F.H. and M.W analyzed the data. F.H. and M.W wrote the paper.

Funding: This research was funded by the R \& D Special Fund for Public Welfare Industry (Agriculture) of China (grant number 201303025) and the Natural Science Foundation of China (grant number 31401690, 31772212).

Conflicts of Interest: The authors declare that there is no conflict of interest.

\section{References}

1. Elad, Y.; Pertot, I.; Marina, A.; Prado, A.M.; Stewart, A. Plant Hosts of Botrytis spp. In Botrytis-The Fungus, the Pathogen and Its Management in Agricultural Systems; Fillinger, S., Elad, Y., Eds.; Springer: Cham, Switzerland, 2016; pp. 413-486.

2. Hao, F.M.; Zhou, Z.L.; Wu, M.D.; Li, G.Q. Molecular characterization of a novel endornavirus from the phytopathogenic fungus Botrytis cinerea. Arch. Virol. 2017, 162, 313-316. [CrossRef] [PubMed]

3. Hao, F.M.; Ding, T.; Wu, M.D.; Zhang, J.; Yang, L.; Chen, W.D.; Li, G.Q. Two novel hypovirulence-associated mycoviruses in the phytopathogenic fungus Botrytis cinerea: Molecular characterization and suppression of infection cushion formation. Viruses 2018, 10, 254. [CrossRef] [PubMed]

4. Wu, M.D.; Zhang, J.; Yang, L.; Li, G.Q. RNA mycoviruses and their role in Botrytis Biology. In Botrytis—The Fungus, the Pathogen and Its Management in Agricultural Systems; Fillinger, S., Elad, Y., Eds.; Springer: Cham, Switzerland, 2016; pp. 71-90.

5. Pearson, M.N.; Bailey, A.M. Viruses of botrytis. Arch. Virol. 2013, 86, 249-272.

6. Yu, L.; Sang, W.; Wu, M.D.; Zhang, J.; Yang, L.; Zhou, Y.J.; Chen, W.D.; Li, G.Q. Novel hypovirulence-associated RNA mycovirus in the plant pathogenic fungus Botrytis cinerea. Appl. Environ. Microbiol. 2015, 81, 2299-2310. [CrossRef] [PubMed]

7. Donaire, L.; Rozas, J.; María, A. Molecular characterization of Botrytis ourmia-like virus, a mycovirus close to the plant pathogenic genus Ourmiavirus. Virology 2016, 489, 158-164. [CrossRef] [PubMed]

8. Donaire, L.; Pagan, I.; Ayllon, M.A. Characterization of Botrytis cinerea negative-stranded RNA virus 1, a new mycovirus related to plant viruses, and a reconstruction of host pattern evolution in negative-sense ssRNA viruses. Virology 2016, 499, 212-218. [CrossRef] [PubMed]

9. Easton, A.J.; Pringle, C.R. Order Mononegavirales. In Virus Taxonomy: Classification and Nomenclature of Viruses, Ninth Report of the International Committee on Taxonomy of Viruses; King, M.Q., Adams, M.J., Carstens, E.B., Lefkowitz, E.J., Eds.; Elsevier, Academic Press: London, UK, 2011; pp. 653-657.

10. Dietzgen, R.G.; Kondo, H.; Goodin, M.M.; Kurath, G.; Vasilakis, N. The family Rhabdoviridae: mono- and bipartite negative-sense RNA viruses with diverse genome organization and common evolutionary origins. Virus Res. 2017, 227, 158-170. [CrossRef] [PubMed]

11. Kondo, H.; Chiba, S.; Toyoda, K.; Suzuki, N. Evidence for negative-strand RNA virus infection in fungi. Virology 2013, 435, 201-209. [CrossRef] [PubMed]

12. Afonso, C.L.; Amarasinghe, G.K.; Bányai, K.; Bao, Y.; Basler, C.F.; Bavari, S.; Bejerman, N.; Blasdell, K.R.; Briand, F.; Briese, T.; et al. Taxonomy of the order Mononegavirales: Update. 2016. Arch. Virol. 2016, 161, 2351-2360. [CrossRef] [PubMed]

13. Walker, P.J.; Firth, C.; Widen, S.G.; Blasdell, K.R.; Guzman, H.; Wood, T.G.; Paradkar, P.N.; Holmes, E.C.; Tesh, R.B.; Vasilakis, N. Evolution of genome size and complexity in the Rhabdoviridae. PLoS Pathog. 2015, 11, 1-25. [CrossRef] [PubMed]

14. Liu, L.; Xie, J.; Cheng, J.; Fu, Y.; Li, G.; Yi, X.; Jiang, D. Fungal negative-stranded RNA virus that is related to bornaviruses and nyaviruses. Proc. Natl. Acad. Sci. USA 2014, 111, 12205-12210. [CrossRef] [PubMed]

15. Marzano, S.Y.; Domier, L.L. Novel mycoviruses discovered from metatranscriptomics survey of soybean phyllosphere phytobiomes. Virus Res. 2016, 213, 332-342. [CrossRef] [PubMed] 
16. Marzano, L.S.Y.; Nelson, B.D.; Ajayi-Oyetunde, O.; Bradley, C.A.; Hughes, T.J.; Hartman, G.L.; Eastburn, D.M.; Domiera, L.L. Identification of diverse mycoviruses through metatranscriptomics characterization of the viromes of five major fungal plant pathogens. J. Virol. 2016, 90, 6846-6863. [CrossRef] [PubMed]

17. Wang, L.; He, H.; Wang, S.; Chen, X.; Qiu, D.; Kondob, H.; Guo, L. Evidence for a novel negative-stranded RNA mycovirus isolated from the plant pathogenic fungus Fusarium graminearum. Virology 2018, 518, 232-240. [CrossRef] [PubMed]

18. Mu, F.; Xie, J.T.; Cheng, S.F.; You, M.P.; Barbetti, M.J.; Jia, J.C.; Wang, Q.Q.; Cheng, J.S.; Fu, Y.P.; Chen, T.; et al. Virome characterization of a collection of Sclerotinia sclerotiorum from Australia. Front. Microbial. 2018, 8, 2540. [CrossRef] [PubMed]

19. Wu, M.D.; Zhang, L.; Li, G.Q. Genome characterization of a debilitation-associated mitovirus infecting the phytopathogenic fungus Botrytis cinerea. Virology 2010, 406, 117-126. [CrossRef] [PubMed]

20. Wu, M.D.; Zhang, L.; Li, G.Q.; Jiang, D.H.; Hou, M.S.; Huang, H.C. Hypovirulence and double-stranded RNA in Botrytis cinerea. Phytopathology 2007, 97, 1590-1599. [CrossRef] [PubMed]

21. Wu, M.D.; Jin, F.Y.; Zhang, J.; Yang, L.; Jiang, D.H.; Li, G.Q. Characterization of a novel bipartite double-stranded RNA mycovirus conferring hypovirulence in the phytopathogenic fungus Botrytis porri. J. Virol. 2012, 86, 6605-6619. [CrossRef] [PubMed]

22. Kumar, S.; Stecher, G.; Tamura, K. MEGA7: Molecular Evolutionary Genetics Analysis version 7.0 for bigger datasets. Mol. Blol. Evol. 2016, 33, 1870-1874. [CrossRef] [PubMed]

23. Krogh, A.; Larsson, B.; von Heijne, G.; Sonnhammer, E.L. Predicting transmembrane protein topology with a hidden Markov model: Application to complete genomes. J. Mol. Biol. 2001, 305, 567-580. [CrossRef] [PubMed]

24. Choi, G.H.; Nuss, D.L. Hypovirulence of chestnut blight fungus conferred by an infectious viral cDNA. Science 1992, 257, 800-803. [CrossRef] [PubMed]

25. Van Diepeningen, A.D.; Debets, A.J.M.; Hoekstra, R.F. Dynamics of dsRNA mycoviruses in black Aspergillus populations. Fungal. Genet. Biol. 2006, 43, 446-452. [CrossRef] [PubMed]

26. Kim, J.M.; Jung, J.E.; Park, J.A.; Park, S.M.; Cha, B.J.; Kim, D.H. Biological function of a novel chrysovirus, CnV1-BS122, in the Korean Cryphonectria nitschkei BS122 strain. J. Biosci. Bioeng. 2013, 115, 1-3. [CrossRef] [PubMed]

27. Fulbright, D.W. Effect of eliminating dsRNA in hypovirulent Endothia parasitica. Phytopathology 1984, 74, 722-724. [CrossRef]

28. Elias, K.S.; Cotty, P.J. Incidence and stability of infection by double-stranded RNA genetic elements in Aspergillus section Flavi and effects on aflatoxigenicity. Can. J. Bot. 1996, 74, 716-725. [CrossRef]

29. Kwon, Y.C.; Jeong, D.W.; Gim, S.I.; Ro, H.S.; Lee, H.S. Curing viruses in Pleurotus ostreatus by growth on a limited nutrient medium containing cAMP and rifamycin. J. Virol. Methods 2012, 185, 156-159. [CrossRef] [PubMed]

30. Amselem, J.; Cuomo, C.A.; van Kan, J.A.L.; Viaud, M.; Benito, E.P.; Couloux, A.; Coutinho, P.M.; de Vries, R.P.; Dyer, P.S.; Fillinger, S.; et al. Genomic analysis of the necrotrophic fungal pathogens Sclerotinia sclerotiorum and Botrytis cinerea. PLoS Genet. 2011, 7, e1002230. [CrossRef] [PubMed]

31. Bolton, M.D.; Thomma, B.P.; Nelson, B.D. Sclerotinia sclerotiorum (Lib.) de Bary: Biology and molecular traits of a cosmopolitan pathogen. Mol. Plant. Pathol. 2006, 7, 1-16. [CrossRef] [PubMed]

32. Coenen, A.; Kevei, F.; Hoekstra, R.F. Factors affecting the spread of double-stranded RNA viruses in Aspergillus nidulans. Genet. Res. 1997, 69, 1-10. [CrossRef] [PubMed]

33. Melzer, M.S.; Ikeda, S.S.; Boland, G.J. Interspecific transmission of double-stranded RNA and hypovirulence from Sclerotinia sclerotiorum to S. minor. Phytopathology 2002, 92, 780-784. [CrossRef] [PubMed]

34. Liu, Y.C.; Hillman, B.I.; Linder-Basso, D.; Kaneko, S.; Milgroom, M.G. Evidence for interspecies transmission of viruses in natural populations of filamentous fungi in the genus Cryphonectria. Mol. Ecol. 2003, 12, 1619-1628. [CrossRef] [PubMed]

35. Bouneb, M.; Turchetti, T.; Nannelli, R.; Roversi, P.F.; Paoli, F.; Danti, R.; Simoni, S. Occurrence and transmission of mycovirus Cryphonectria hypovirus 1 from dejecta of Thyreophagus corticalis (Acari, Acaridae). Fungal Biol. 2016, 120, 351-357. [CrossRef] [PubMed]

36. Liu, S.; Xie, J.; Cheng, J.; Li, B.; Chen, T.; Fu, Y. Fungal DNA virus infects a mycophagous insect and utilizes it as a transmission vector. Proc. Natl. Acad. Sci. USA 2016, 113, 12803-12808. [CrossRef] [PubMed] 
37. Korinsak, S.; Tangphatsornruang, S.; Pootakham, W.; Wanchana, S.; Plabpla, A.; Jantasuriyarat, C.; Patarapuwadol, S.; Vanavichit, A.; Toojinda, T. Genome-wide association mapping of virulence gene in rice blast fungus Magnaporthe oryzae using a genotyping by sequencing approach. Genomics 2018. [CrossRef] [PubMed]

38. Castiblanco, V.; Marulanda, J.J.; Würschum, T.; Miedaner, T. Candidate gene based association mapping in Fusarium culmorum for field quantitative pathogenicity and mycotoxin production in wheat. BMC Genet. 2017, 18, 49. [CrossRef] [PubMed]

39. Rodríguez-García, C.; Medina, V.; Alonso, A.; Ayllón, M.A. Mycoviruses of Botrytis cinerea isolates from different hosts. Ann. Appl. Biol. 2013, 164, 46-61. [CrossRef]

2018 by the authors. Licensee MDPI, Basel, Switzerland. This article is an open access article distributed under the terms and conditions of the Creative Commons Attribution (CC BY) license (http://creativecommons.org/licenses/by/4.0/). 